\title{
XMCD Study of the Magnetic Exchange Coupling in a Fluoride-Bridged Dy-Cr Molecular Cluster
}

\author{
Jan Dreiser, ${ }^{*}$ Cinthia Piamonteze and Frithjof Nolting \\ Swiss Light Source, Paul Scherrer Institut, CH-5232 Villigen PSI, Switzerland \\ Kasper S. Pedersen and Jesper Bendix \\ Department of Chemistry, University of Copenhagen, DK-2100 Copenhagen, Denmark \\ Stefano Rusponi and Harald Brune \\ Institute of Condensed Matter Physics, Ecole Polytechnique Fédérale de Lausanne, CH-1015 Lausanne, Switzerland
}

(Received 3 June 2012, in final form 23 October 2012)

\begin{abstract}
We have studied the fluoride-bridged Dy-Cr molecular nanomagnet [Dy(hfac $)_{4^{-}}$ $\mathrm{CrF}_{2}$ (py) $\left.)_{4}\right] \cdot 1 / 2 \mathrm{CHCl}_{3}$ by x-ray magnetic circular dichroism (XMCD). The obtained element-specific magnetization curves allow for a quantification of the sign and strength of the magnetic exchange coupling between the Dy and the $\mathrm{Cr}$ ions. In an effective spin- $1 / 2$ formalism only taking into account the ground Kramers doublet of the Dy ${ }^{\mathrm{III}}$ ion, we find a coupling strength of $j_{\mathrm{eff}, z}=$ $-2.3(1) \mathrm{cm}^{-1}$. Further, we find that the ground Kramers doublet is nearly perfectly axial with $g_{\text {eff }, z, \text { Dy }}=19.6(6)$ and $g_{\text {eff }, x y, \text { Dy }}=0(2)$. The coupling value corresponds to a "true", non-effective isotropic coupling of $j=-0.16 \mathrm{~cm}^{-1}$ when taking into account a full $J=15 / 2$ angular momentum. This coupling strength is comparable to that of $j=-0.18 \mathrm{~cm}^{-1}$ previously found in the related fluoride-bridged compound Dy-Cr-Dy.
\end{abstract}

PACS numbers: 75.50.Xx, 78.70.Dm, 75.30.Et

Keywords: Molecular Nanomagnets, X-ray magnetic circular dichroism, Exchange Coupling, Rare Earths DOI: $10.3938 / \mathrm{jkps} .62 .1368$

\section{INTRODUCTION}

In recent years molecular nanomagnets $[1,2]$ (MNMs) have gained considerable attention due to their potential for applications in molecular spintronics [3], quantum information processing [4] and molecular coolers [5]. While at early times most of the MNMs were based on $3 d$ transition metal ions, research has lately been extended to compounds containing rare earth $(4 f)$ ions [6]. The latter have rather different magnetic properties, which are mainly determined by strong spin-orbit coupling dominating over the ligand field mostly resulting in significant orbital contributions to their magnetic moments and large anisotropy splittings. Despite these interesting properties, magnetic exchange coupling involving $4 f$ ions turns out to be rather weak because of the protected nature of the $4 f$ shell. Understanding the magnetic behavior of $4 f$-containing MNMs is more challenging than that of many $3 d$ compounds because of the large number of parameters describing the ligand field, even for rather high symmetries. The low-temperature behavior

\footnotetext{
*E-mail: jan.dreiser@psi.ch
}

of the $\chi \cdot T$ product, where $\chi$ is the magnetic susceptibility, is frequently used to deduce the sign and strength of an intracluster exchange coupling. However, the large anisotropy splittings of $4 f$ ions typically extending up to several hundreds of $\mathrm{cm}^{-1}$ can themselves modify the $\chi \cdot T$ curve, even in the absence of exchange coupling. Therefore, techniques complementary to the widespread SQUID magnetometry are necessary in order to obtain precise values of the $3 d-4 f$ exchange coupling. Recently, electron paramagnetic resonance [7] and inelastic neutron scattering [8] have been used to extract the exchange coupling strengths. Furthermore, x-ray magnetic circular dichroism [9] (XMCD) is suitable for the quantification of intracluster $3 d-4 f$ exchange coupling as shown in Ref. 10 where we have investigated a fluoride-bridged Dy-Cr-Dy trimer.

There is only a small number of reported $3 d-4 f$ MNMs containing fluoride bridges between paramagnetic units $[10,11]$, although fluoride bridges are quite attractive because of their tendency towards formation of pseudolinear bridges and hence the possibility of predictable cluster topologies. The reason for the scarcity of fluoridebridged $3 d-4 f$ MNMs lies in the challenge of their synthesis which is complicated by the possible formation of 


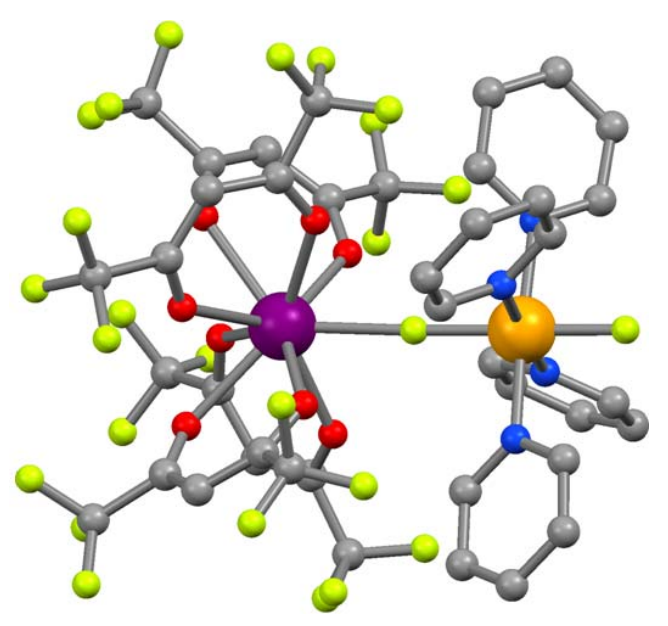

Fig. 1. (Color online) Molecular structure of the investigated dinuclear compound $\left[\mathrm{Dy}(\mathrm{hfac})_{4}-\mathrm{CrF}_{2}(\mathrm{py})_{4}\right]$. The solvent molecule and $\mathrm{H}$ atoms have been omitted for clarity. Color code: purple: Dy; orange: Cr; light green: F; gray: C; red: O; blue: N.

insoluble lanthanide(III) fluoride.

In this work, we use XMCD to obtain element-specific magnetization curves $M(H)$ of the fluoride-bridged MNM $\left[\mathrm{Dy}(\mathrm{hfac})_{4}-\mathrm{CrF}_{2}(\mathrm{py})_{4}\right] \cdot 1 / 2 \mathrm{CHCl}_{3}$ (in short: Dy$\mathrm{Cr}$ ), with hfacH $=1,1,1,5,5,5$-hexafluoroacetylacetone, py $=$ pyridine). In such clusters exchange coupling leads to a modification of the element-specific magnetization curves with respect to the case of uncoupled ions in a ligand field. In the following, we will exploit this effect to quantify the magnetic exchange coupling between ions by fitting a spin-Hamiltonian model to the XMCD data.

\section{EXPERIMENTS AND DISCUSSION}

Experiments have been performed on a powder sample of Dy-Cr. This compound has been obtained very recently [10] along with a related trinuclear species. Its molecular structure is shown in Fig. 1. X-ray absorption spectra (XAS) were measured at the X-Treme beamline [12] (Swiss Light Source, Paul Scherrer Institut, Switzerland) in total electron yield (TEY) mode. For all measurements the temperature was $2.0 \mathrm{~K}$, and magnetic fields of up to $\mu_{0} H_{x}= \pm 6$ Tesla along the beam direction were applied. The beam was defocused $\left(\sim 1 \mathrm{~mm}^{2}\right.$ diameter) and the flux was kept very low. Energy scans were recorded on-the-fly, that is, the monochromator and insertion device were moving continuously while the data was acquired [13]. One XMCD spectrum corresponds to the difference between XAS spectra obtained for the two circular polarizations, using a sequence of 9 XAS scans. Magnetization curves were obtained by measuring the TEY at the energy of maximum dichroism and at the preedge in an alternating fashion while ramping the magnetic field. To obtain absolute values of the magne-
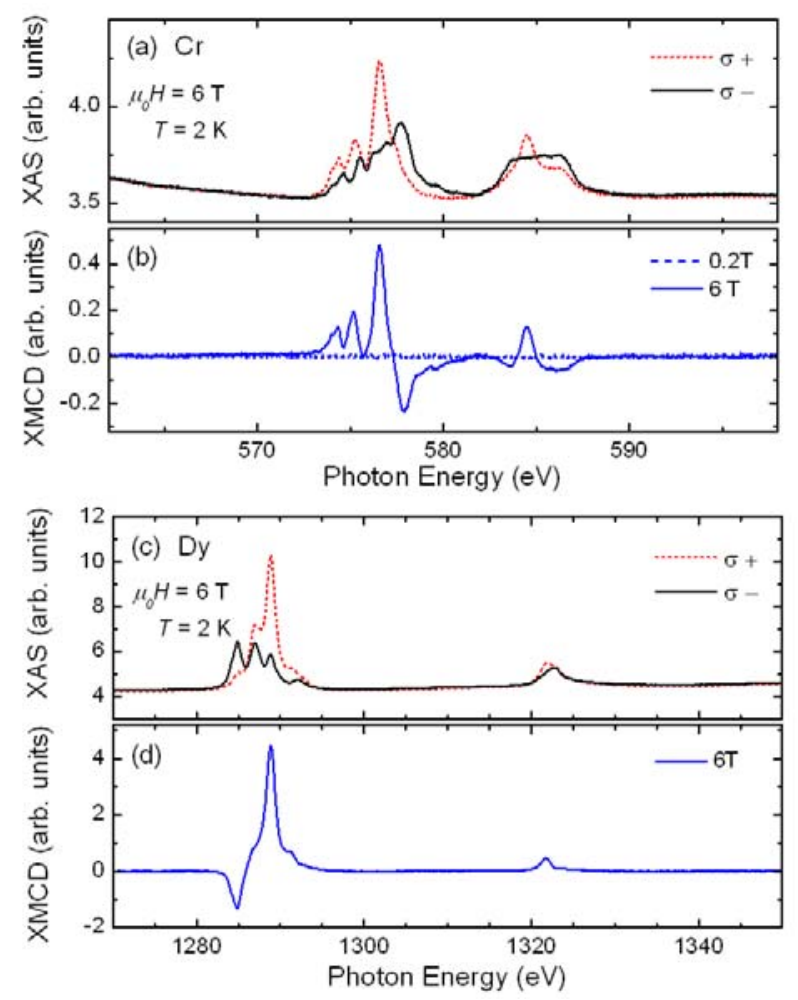

Fig. 2. (Color online) (a), (c) Polarization-dependent xray absorption spectra recorded at the $\mathrm{Cr}_{2,3}$ and $\mathrm{Dy} \mathrm{M}_{4,5}$ edges. (b), (d) Corresponding XMCD spectra.

tization, sum rule analysis [14] was performed for the Dy $\mathrm{x}$-ray spectra, while this was not possible for $\mathrm{Cr}$, since for the latter the separation between $\mathrm{L}_{2,3}$ edges is too small [15].

Absorption and dichroism spectra measured in magnetic field at the $\mathrm{Cr} \mathrm{L}_{2,3}$ edges and at the Dy $\mathrm{M}_{4,5}$ edges are shown in Figs. 2(a), (b) and (c), (d), respectively. At $6 \mathrm{~T}$, there is clearly a strong dichroic response for both $\mathrm{Cr}^{\mathrm{III}}$ and $\mathrm{Dy}^{\mathrm{III}}$ ions. However, at $0.2 \mathrm{~T}$ there is virtually no $\mathrm{Cr}$ dichroism, indicating that the net $\mathrm{Cr}$ magnetic moment along the magnetic field direction is zero. This observation can also be made in the $\mathrm{Cr}$ magnetization curve as shown in Fig. 3(a). Here, a wiggle is seen at around zero field, while at stronger fields the curve resembles that of a paramagnetic ion. In contrast, the wiggle is absent in the Dy magnetization curve plotted in Fig. 3(b). It quickly reaches saturation at rather small fields of approximately $2 \mathrm{~T}$, suggesting a large magnetic moment. Absolute values of the total (spin and orbital) Dy magnetic moments extracted from sum rule analysis performed on x-ray spectra measured at various magnetic fields are shown as dark blue squares in Fig. 3(b). In the analysis, a Landé $g$-factor of $4 / 3$ for the Dy ${ }^{\mathrm{III}}$ ion and fully polarized x-rays were taken into account, which defines the right-hand $y$-scale of absolute magnetic moment. In contrast the absolute scale of $\mathrm{Cr}$ magnetization in Fig. 3(a) was imposed by matching a saturation value 


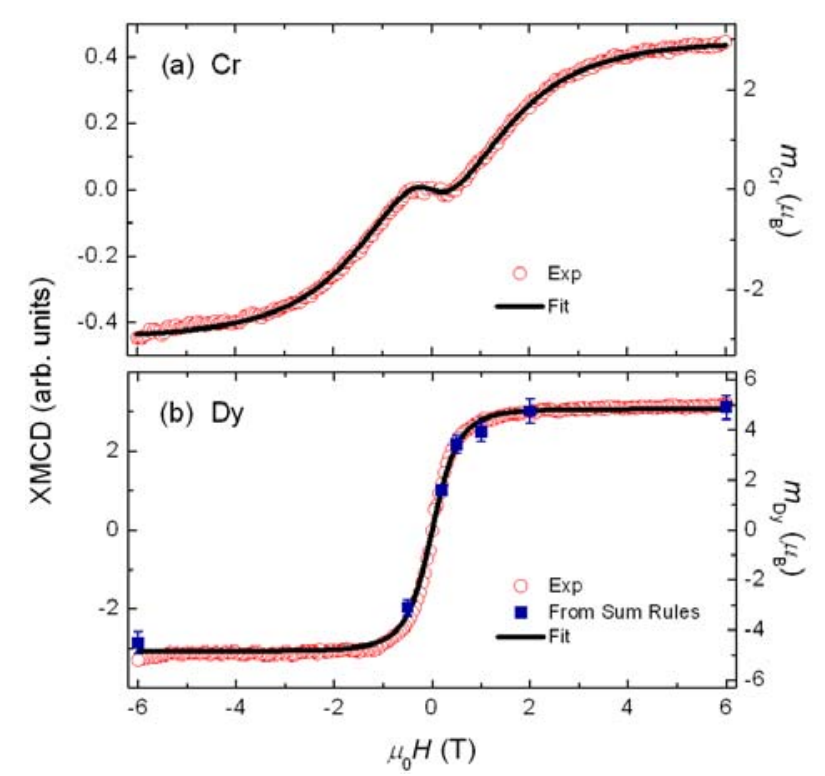

Fig. 3. (Color online) Element-specific magnetization curves obtained from polarization-dependent x-ray absorption at $2 \mathrm{~K}$. Blue squares represent the absolute magnetic moments obtained from sum rule analysis and solid lines are fits to the data using the model and best-fit parameters given in the text.

\section{of $3 \mu_{B}$ consistent with high-spin $\mathrm{Cr}^{\mathrm{III}}$.}

The wiggle shape in Fig. 3(a) resembles that seen in the $\mathrm{Cr}$ magnetization curve of the related Dy-Cr-Dy trimer investigated in Ref. 10, albeit it appears less pronounced. It is the signature of a weak antiferromagnetic coupling between Dy and Cr. In order to perform a quantitative investigation, we have used a spin-Hamiltonian model describing the Dy-Cr compound. While the $\mathrm{Cr}^{\mathrm{III}}$ ion is described by a spin $S=3 / 2$ without significant orbital contribution, the treatment of the Dy ${ }^{\mathrm{III}}$ ion needs some approximations to avoid overparameterization of the model. According to Hund's rules the ground state multiplet of $\mathrm{Dy}{ }^{\mathrm{III}}$ is $J=15 / 2$, and the ligand field leads to a splitting of the resulting 16 states into Kramers doublets each of them being at least doubly degenerate. Since the separations between the Kramers doublets are typically much larger than $2 \mathrm{~K}$, we have used the widespread approximation of taking into account the lowest Kramers doublet only, which is mapped onto an effective or pseudospin of $\tau=1 / 2$. The anisotropy of the $\mathrm{Dy}^{\mathrm{III}}$ ion then results in an anisotropic exchange coupling $j$ and an anisotropic $\mathbf{g}_{\text {eff, Dy-matrix. Hence, the }}$ spin Hamiltonian describing the Cr-Dy MNM reads

$$
\hat{H}=-\hat{\tau}_{\text {Dy }} \mathbf{j}_{\text {eff }} \hat{\mathbf{S}}_{\mathrm{Cr}}+\mu_{B}\left(\mathbf{g}_{\text {eff, Dy }} \hat{\tau}_{\text {Dy }}+g_{\mathrm{Cr}} \hat{\mathbf{S}}_{\mathrm{Cr}}\right) \cdot \mathbf{B}
$$

Here, the first term describes the magnetic exchange coupling and the second term takes into account the interaction with magnetic field. $\hat{\mathbf{S}}_{\mathrm{Cr}}$ and $\hat{\tau}_{\text {Dy }}$ are the spin and pseudospin operators of the $\mathrm{Cr}^{\mathrm{III}}$ and $\mathrm{Dy}^{\mathrm{III}}$ ions. In the case of a purely axial ground doublet, that is, if the ground Kramers doublet is an eigenstate of the $\hat{J}_{z}$ oper- ator, the $\mathbf{j}_{\text {eff }}$ and $\mathbf{g}_{\text {eff,Dy }}$ matrices have only a single entry $\mathbf{j}_{\text {eff }}=\operatorname{diag}\left(0,0, j_{z}\right)$ and $\mathbf{g}_{\text {eff,Dy }}=\operatorname{diag}\left(0,0, g_{\mathrm{eff}, z}\right)$. More details can be found e.g. in the Supporting Information of Ref. 10.

We have performed least-squares fits to the experimental element-specific magnetization curves, based on full diagonalization of the spin-Hamiltonian Eq. (1). The powder average of the magnetization curves was achieved using a 16-point Lebedev-Laikov grid [16]. In the fits, the parameters $j_{\mathrm{eff}, z}$ and $g_{\mathrm{eff}, z, \text { Dy }}$ were freely varied, while the Cr $g$-factor was fixed to $g_{\mathrm{Cr}}=2.0$.

We obtain the best-fit values

$$
\begin{aligned}
& j_{\mathrm{eff}, z}=-2.3(1) \mathrm{cm}^{-1} \\
& g_{\mathrm{eff}, z, \text { Dy }}=19.6(6),
\end{aligned}
$$

and the corresponding best-fit curves are shown as solid black lines in Fig. 3, demonstrating excellent agreement with the experimental data. Introducing additional transverse elements in the Dy g-matrix $\mathbf{g}_{\text {eff }}=$ $\operatorname{diag}\left(g_{\mathrm{eff}, x y}, g_{\mathrm{eff}, x y}, g_{\mathrm{eff}, z}\right)$ did not improve the fits and resulted in $g_{x y}=0$ while the other best-fit parameters given above remained unchanged. The observed $\mathbf{g}_{\text {eff,Dy }}{ }^{-}$ anisotropy indicates a strongly axial ground Kramers doublet, and the $g_{z}$ value is very close to 20.0 which is expected for a ground doublet of $m_{J, G S}= \pm 15 / 2$ (because of $m_{J, G S}=g_{\mathrm{eff}, z} /\left(2 g_{J}\right)$ in first order perturbation theory, as shown in the Supporting Information of Ref. 10). The assumption of a fully isotropic $\mathrm{Dy}^{\mathrm{III}}$ angular momentum of $J=15 / 2$ is incompatible with our data, since in that case the saturation value of the Dy ${ }^{\mathrm{III}}$ magnetic moment would be $10 \mu_{B}$ instead of approximately $5 \mu_{B}$ which is observed. The drop of the saturation moment originates from the powder average over a strongly anisotropic system and is well reproduced in our numerical calculations. In our recent study Ref. 10 we found that a first-order approximation of the "true" Dy-Cr coupling $j$, meaning the non-effective coupling in a full $J=15 / 2$ spin-Hamiltonian, can be obtained by $j=j_{\mathrm{eff}, z} \cdot g_{J} / g_{\mathrm{eff}, z}$, leading to $j=-0.16 \mathrm{~cm}^{-1}$. This value is comparable to that found for the related DyCr-Dy trimer studied in Ref. 10, where the coupling was determined to be $j=-0.18 \mathrm{~cm}^{-1}$, consistent with the approximately equal Dy-F and Cr-F bond lengths in the two compounds. In contrast, the zero-crossing fields of the wiggle are considerably smaller here than in the DyCr-Dy trimer. This implies that a smaller magnetic field is sufficient to reorient the $\mathrm{Cr}$ magnetic moment in the dinuclear compound, which is expected even for identical coupling strength because of the reduced exchange coupling energy as compared to the trinuclear counterpart.

\section{CONCLUSION}

In summary, we have performed a low-temperature high-magnetic field XMCD study on a powder sample of the dinuclear molecular nanomagnet $\left[\mathrm{Dy}(\mathrm{hfac})_{4^{-}}\right.$ 
$\left.\mathrm{CrF}_{2}(\mathrm{py})_{4}\right] \cdot 1 / 2 \mathrm{CHCl}_{3}$ in which a $\mathrm{Dy}^{\mathrm{III}}$ and a $\mathrm{Cr}^{\mathrm{III}}$ ion are exchange coupled via a fluoride bridge. The obtained element-specific magnetization curves are modified with respect to those of free paramagnetic ions by the effect of anisotropy and exchange coupling. Importantly, the latter induces a wiggle shape in the $\mathrm{Cr}$ magnetization curve which indicates a weak antiferromagnetic coupling. Using a spin-Hamiltonian parameterization with an effective spin $\tau=1 / 2$ for the $\mathrm{Dy}^{I I I}$ ion and $S=3 / 2$ for $\mathrm{Cr}^{\mathrm{III}}$ we obtain an effective magnetic exchange coupling of $j_{\text {eff }, z}=-2.3(1) \mathrm{cm}^{-1}$. The Dy ground Kramers doublet is well described by effective, anisotropic $g$-factors $g_{\mathrm{eff}, z \text {,Dy }}$ $=19.6(6)$ and $g_{\text {eff }, x y \text {,Dy }}=0(2)$. From the effective coupling value $j_{\text {eff, } z}$, a non-effective isotropic coupling of $j=$ $-0.16 \mathrm{~cm}^{-1}$ can be deduced for a full $J=15 / 2$ angular momentum description of the Dy ${ }^{\mathrm{III}}$ ion. This coupling strength is comparable to that of $j=-0.18 \mathrm{~cm}^{-1}$ previously found in the related fluoride-bridged compound Dy-Cr-Dy.

\section{ACKNOWLEDGMENTS}

We acknowledge M. Schmidt for technical support. The XMCD measurements have been performed at the X-Treme beamline of the Swiss Light Source, Paul Scherrer Institut, Villigen, Switzerland. We gratefully acknowledge financial support for the XMCD endstation from Ecole Polytechnique Fédérale de Lausanne and from the Swiss National Science Foundation.

\section{REFERENCES}

[1] O. Kahn, in Molecular Magnetism (Wiley-VCH, Weinheim, Germany, 1993).

[2] D. Gatteschi, R. Sessoli and J. Villain, in Molecular Nanomagnets (Oxford University Press, 2006).

[3] A. R. Rocha, V. M. Garcia-suarez, S. W. Bailey, C. J. Lambert, J. Ferrer and S. Sanvito, Nat. Mater. 4, 335 (2005); L. Bogani and W. Wernsdorfer, Nat. Mater. 7, 179 (2008).

[4] M. N. Leuenberger and D. Loss, Nature 410, 789 (2001).

[5] F. Torres, J. M. Hernandez, X. Bohigas and J. Tejada, Appl. Phys. Lett. 77, 3248 (2000); R. Sessoli, Angew. Chem. Int. Ed. 51, 43 (2012).
[6] R. E. P. Winpenny, Chem. Soc. Rev. 27, 447 (1998); R. Sessoli and A. K. Powell, Coord. Chem. Rev. 253, 2328 (2009).

[7] A. Figuerola, V. Tangoulis and Y. Sanakis, Chem. Phys. 334, 204 (2007); A. Okazawa, T. Nogami, H. Nojiri and T. Ishida, Inorg. Chem. 47, 9763 (2008); L. Sorace, C. Sangregorio, A. Figuerola, C. Benelli and D. Gatteschi, Chem. Eur. J. 15, 1377 (2009).

[8] H. U. Güdel, A. Furrer and H. Blank, Inorg. Chem. 29, 4081 (1990); M. A. Aebersold, H. U. Güdel, A. Hauser, A. Furrer, H. Blank and R. Kahn, Phys. Rev. B 48, 12723 (1993).

[9] G. van der Laan and B. T. Thole, Phys. Rev. B 43 , 13401 (1991); J. Stöhr, J. Magn. Magn. Mater. 200, 470 (1999); T. Funk, A. Deb, S. J. George, H. Wang and S. P. Cramer, Coord. Chem. Rev. 249, 3 (2005).

[10] J. Dreiser, K. S. Pedersen, C. Piamonteze, S. Rusponi, Z. Salman, M. E. Ali, M. Schau-Magnussen, C. A. Thuesen, S. Piligkos, H. Weihe, H. Mutka, O. Waldmann, P. Oppeneer, J. Bendix, F. Nolting and H. Brune, Chem. Sci. 3, 1024 (2012).

[11] A. McRobbie, A. R. Sarwar, S. Yeninas, H. Nowell, M. L. Baker, D. Allan, M. Luban, C. A. Muryn, R. G. Pritchard, R. Prozorov, G. A. Timco, F. Tuna, G. F. S. Whitehead and R. E. P. Winpenny, Chem. Commun. 47, 6251 (2011); T. Birk, K. S. Pedersen, C. A. Thuesen, T. Weyhermüller, M. Schau-Magnussen, S. Piligkos, H. Weihe, S. Mossin, M. Evangelisti and J. Bendix, Inorg. Chem. 51, 5435 (2012); C. Aa. Thuesen, K. S. Pedersen, M. Schau-Magnussen, M. Evangelisti, J. Vibenholt, S. Piligkos, H. Weihe and J. Bendix, Dalton Trans. 41, 11284 (2012)

[12] C. Piamonteze, U. Flechsig, S. Rusponi, J. Dreiser, J. Heidler, M. Schmidt, R. Wetter, T. Schmidt, H. Pruchova, J. Krempasky, C. Quitmann, H. Brune, F. Nolting, J. Synchrotron Rad. 19, 661 (2012).

[13] J. Krempasky, U. Flechsig, T. Korhonen, D. Zimoch, C. Quitmann and F. Nolting, AIP Conf. Proc. 1234, 705 (2010).

[14] B. T. Thole, P. Carra, F. Sette and G. van der Laan, Phys. Rev. Lett. 68, 1943 (1992); P. Carra, B. T. Thole, M. Altarelli and X. Wang, Phys. Rev. Lett. 70, 694 (1993).

[15] Y. Teramura, A. Tanaka and T. Jo, J. Phys. Soc. Jpn. 65, 1053 (1996); C. Piamonteze, P. Miedema and F. M. F. de Groot, Phys. Rev. B 80, 184410 (2009).

[16] V. I. Lebedev and D. N. Laikov, Dokl. Math. 366, 741 (1999). 